\title{
Quality improvement project to decrease unnecessary investigations in infants with bronchiolitis in Cork University Hospital
}

\author{
Lizeri Jansen (D) , ${ }^{1,2}$ Gideon-Phil Meyer (D) , ${ }^{1,2}$ Glenn Curtin (D) , ${ }^{1}$ Bryan Lynch, ${ }^{1}$ \\ Rory O'Brien (1) ${ }^{1}$
}

To cite: Jansen L, Meyer GP, Curtin G, et al. Quality improvement project to decrease unnecessary investigations in infants with bronchiolitis in Cork University Hospital. BMJ Open Quality 2021;10:e001428. doi:10.1136/ bmjoq-2021-001428

- Additional supplemental material is published online only. To view, please visit the journal online (http://dx.doi.org/10. 1136/bmjoq-2021-001428).

LJ and G-PM are joint first authors.

Received 27 February 2021 Accepted 25 0ctober 2021

Check for updates

(C) Author(s) (or their employer(s)) 2021. Re-use permitted under CC BY-NC. No commercial re-use. See rights and permissions. Published by BMJ.

${ }^{1}$ Emergency Department, Cork University Hospital, Cork, Ireland ${ }^{2}$ University of Manchester, Manchester, UK

Correspondence to Dr Lizeri Jansen; lizerijansen@gmail.com

\section{ABSTRACT}

Background Bronchiolitis is a common reason for infants to present to the emergency department (ED). Clear evidencebased guidelines exist that recommend against routine radiological and laboratory investigations in this cohort. Despite this, preintervention audit showed that children below 12 months of age with bronchiolitis in the ED during November 2018-January 2019 were receiving unnecessary investigations. Our aim was to improve patient care by decreasing unnecessary investigations in bronchiolitis infants. Methods Baseline assessment comprised a preintervention audit of children less than 12 months of age with a diagnosis of bronchiolitis that presented to ED during November 2018-January 2019. The outcome measure was average weekly hospital length of stay (LOS) process measures were average weekly chest X-ray (CXR) and laboratory investigation rate. The balancing measure was the average weekly representation rate.

Intervention A multimodal intervention was implemented comprising a locally agreed flowchart enhanced by regular feedback on performance using run charts and in-person sessions.

Results A postintervention audit of November 2019January 2020 was undertaken. There was a $57 \%$ reduction in the mean average weekly CXR rate (from $25 \%$ to $11 \%, p$ value 0.009974 significant at $p<0.05$ ); there was an improvement by $56 \%$ in the mean average weekly laboratory investigation rate (from $29 \%$ to $13 \%$, p value 0.005475 , significant at $p<0.05$ ) in the preintervention and postintervention periods, respectively. The mean average weekly representations remained at $4 \%$ preintervention and postintervention ( $p$ value 0.737 ). There was no significant difference in hospital LOS (from 25.3 hours to 20.7 hours, $p$ value 0.270549 )

Conclusion An evidence-based protocol improved physicians' ability in diagnosing and managing infants with bronchiolitis. This led to a reduction in unnecessary and potential harmful investigations, thereby improving patient quality of care. This improvement will contribute to decreased healthcare cost and appropriate use of resources during the high-pressured winter period.

\section{INTRODUCTION}

Problem description

Bronchiolitis is a common presentation of infants in the busy emergency department (ED). ${ }^{1}$
These infants are often exposed to diagnostic interventions that are not evidencebased. Inappropriate management of infants with bronchiolitis results in less appropriate discharges and poor outcomes when admitted. This increases the burden on the ED. There are evidence-based standards of care which, when implemented, will improve quality of care in patients. ${ }^{2}$

A retrospective audit was performed on all cases of infants below 1 year of age, diagnosed with bronchiolitis, every week from 1 November 2018 to 30 January 2019 (see table 1). The results confirmed that a large number of patients had received inappropriate investigations, namely, chest X-rays (CXRs) and blood tests, as a means to diagnose bronchiolitis. A total of 323 patients were diagnosed with bronchiolitis (see table 1), of which a weekly average of $25 \%$ had CXRs and a weekly average of $29 \%$ had blood tests.

\section{Available knowledge}

Bronchiolitis is the most common lower respiratory tract disease in infants below 1 year of age. ${ }^{3}$ The diagnosis is made clinically with no routine requirement for radiological or laboratory investigations. Management is supportive and includes ensuring appropriate fluid intake and respiratory support, as well as minimal handling. ${ }^{4}$ Best practice should be the avoidance of inappropriate and distressing investigations.

CXRs are not beneficial in infants with bronchiolitis as they often mimic changes seen in pneumonia and should therefore not be performed. They may result in inappropriate administration of antibiotics. Routine blood tests or blood gas sampling are not indicated to diagnose or guide treatment of bronchiolitis. ${ }^{3}$

Good supportive management is the standard of care. Clinical assessment of hydration 
Table 1 Patient demographics and summary of results

\begin{tabular}{|c|c|c|c|c|}
\hline & Preintervention & Postintervention & Relative risk reduction & $P$ value \\
\hline Total patients & 323 & 320 & & \\
\hline Male & 182 & 190 & & \\
\hline Mean age (months) & 4 & 5 & & \\
\hline Total blood investigations & $92(28 \%)$ & $43(13 \%)$ & $53 \%$ & $<0.00001$ \\
\hline Total CXRs & $74(23 \%)$ & $41(13 \%)$ & $44 \%$ & 0.00836 \\
\hline CXR, cumulative average rate & $25 \%$ & $11 \%$ & $57 \%$ & 0.009974 \\
\hline Mean hospital length of stay (hours) & 25.3 & 20.7 & $18 \%$ & 0.270549 \\
\hline Total representations & 17 & 15 & $11 \%$ & 0.737 \\
\hline
\end{tabular}

CXR, chest X-ray.

status with appropriate rehydration with nasogastric feeds, if required, is considered best practice. For infants who have impending respiratory failure or deteriorate on nasogastric feeds, intravenous fluids may be considered. ${ }^{3}$ In moderate or severe illness, feeds are best administered at two-thirds of the maintenance volume due to risk of syndrome of inappropriate antidiuretic hormone secretion. ${ }^{4}$ In the event of hypoxia, supplemental oxygen should be administered by nasal prong oxygen if pulse oximetry readings are below $92 \%$. If nasal prongs are ineffective or in cases of severe distress with impending respiratory failure, Continuous Positive Airway Pressure (CPAP) or humidified high-flow nasal prong oxygen should be administered. Oxygen should be titrated to keep oxygenation at $\geq 92 \% .{ }^{56}$ Evidence has shown that the following should not form part of the management of bronchiolitis: antibiotics, hypertonic saline, nebulised epinephrine, salbutamol, montelukast, ipratropium bromide, systemic or inhaled corticosteroids, or combinations of the aforementioned. ${ }^{6}$

There are clinical features of moderate-severe disease that warrant urgent hospital admission. These are apnoea; severe work of breathing (like grunting, marked sternal recession); tachypnoea of $>70$ breaths/min, cyanosis, oxygen saturation below $92 \%$ on room air, reduced feeding below $50 \%$ of normal or evidence of dehydration. ${ }^{36}$ Infants with features of mild bronchiolitis could be treated at home and do not need admission to hospital. It is important to note that infants with certain risk factors are prone to deterioration, and therefore a low threshold for admission should be maintained. These are infants with prematurity, chronic lung disease, cardiac disease, immunodeficiency and neuromuscular diseases. ${ }^{2}$ Infants under 6 weeks of age also tend to deteriorate rapidly, and care should be taken prior to discharge. ${ }^{7}$

A child can be safely discharged home if the following criteria have been met: adequate feeding has been established; oxygen saturation is above $92 \%$ for 4 hours, even during sleep. Certain factors may also affect a carer's capacity to care for a child with bronchiolitis at home, which needs to be considered when assessing whether a child can safely be discharged and may warrant a child to be admitted. ${ }^{6}$ Infants with bronchiolitis that have been ill less than 3 days often worsen on the third and fourth day of illness and may need to represent to the ED. ${ }^{7}$

\section{Rationale}

Stakeholder meetings concluded that the use of unnecessary investigations is likely due to the high patient load and lack of confidence of doctors to withhold investigations in these small babies with respiratory illness.

There was no guideline available to aid in the approach to diagnosis and management that was easy and free to access, yet extensive enough to safely advise the appropriate discharge of some patients and to guide management of more severe cases. There was a need to produce an evidence-based guideline that could meet all the aforementioned criteria to improve care of infants with bronchiolitis.

\section{Specific aims}

The aim of this study was to improve quality of patient care by decreasing the number of inappropriate investigations (namely, X-rays and blood tests) of patients with bronchiolitis below 1 year of age, to below $15 \%$, and hereby decreasing patient hospital length of stay (LOS) without increasing the representation rate. The aim was to be accomplished by implementation of an evidencebased protocol on diagnosis and management of these patients.

\section{METHODS}

\section{Context}

The project took place in Cork University Hospital's ED, a mixed ED that assesses approximately 15000 children per annum, approximately 500 of which have a diagnosis of bronchiolitis. 
The need for a quality improvement project in bronchiolitis was realised by a team of emergency medicine doctors, a registrar and a consultant (LJ and ROB) who recognised a trend of inappropriate investigations in infants with bronchiolitis. They recruited other members of the ED (G-PM, GC and BL) to get involved to assist with data capturing and interpretation before and after the intervention. The project was also introduced to doctors of the paediatric department to ensure compliance with the protocol after care has been taken over by the ward.

The patient population comprised all patients below 1 year of age presenting to the ED who were diagnosed with bronchiolitis by the treating doctor from 1 November 2018 to 30 January 2019 (preintervention before protocol introduction) and from 1 November 2019 to 30 January 2020 (postintervention after protocol introduction).

\section{Interventions}

An evidence-based protocol was created that is easy to read in the form of a flowchart with tick boxes (see online supplemental appendices A,B). It allows doctors to classify patients into either being mild and safe for discharge, or moderate or severe, which warrants admission. Each category of patients has evidence-based management guidelines according to severity of disease. This provides a safety net to the treating physician that no further investigations or treatment is advised according to the current evidence for a patient with bronchiolitis. The protocol was introduced on 1 November 2019. It was made freely available on the hospital guidelines website and printed on posters at the workstations.

Together with the protocol, formal and informal teaching sessions were held with nursing staff, doctors of the ED and doctors of the paediatric department. In addition, email reminders were used to remind staff of the protocol and the best standard of care. This educated the staff on the guideline, emphasised the importance of supportive treatment and educated staff on the insertion and use of nasogastric tubes for feeding instead of intravenous routes for hydration. Education also focused on the use of humidified high-flow nasal prong oxygen. By addressing both the emergency medicine and paediatric teams, there was continuation of evidence-based care after the patient had been referred to the paediatric medical team.

An information leaflet was also designed to inform the caregiver of the diagnosis, the evidence for best treatment practices, and evidence against certain treatments and investigations, and the danger signs to look for when the patient is discharged.

\section{Study of the interventions}

After the introduction of the new protocol, a retrospective review was performed at the end of every 7-day cycle. A retrospective audit was performed to determine the number of patients attending to the ED below 1 year of age with a respiratory-related triage complaint. These files were reviewed and the patients with diagnoses other than bronchiolitis were excluded. An audit was then performed on the number of blood tests performed and the number of CXRs ordered. The number of patients admitted and discharged was also recorded every week. The patient's LOS in the ED was also recorded as per the time from arrival to the ED at the reception on file generation to time to disposition, either discharged off the electronic system when the file is filed away by reception or admitted to the ward by the administrative clerks. The total hospital LOS was also recorded.

The results of the aforementioned weekly retrospective audit were plotted on a run chart, and a meeting was held with the stakeholders to discuss the progress and encourage better compliance through multiple plan-dostudy-act (PDSA) cycles.

\section{Measures}

The Institute for Healthcare Improvement(IHI) framework of quality improvement was used. The primary outcome measure was average hospital LOS. The process measures recorded were the number of CXRs ordered and the number of laboratory investigations performed. A decrease in these would suggest decreased time wasted that could be used for diagnosis and management, and avoidance of potential harm by exposure to radiation and invasive procedures. Representation rate was chosen as the balancing measure.

\section{Analysis}

Fishbone diagrams were used for root cause analysis. A statistical process control chart, together with run charts, was used to track our weekly progress towards our goal. $\chi^{2}$ test statistics was used to calculate the statistical significance of the decrease in the number of CXRs and laboratory investigations.

\section{RESULTS}

During the course of this project, 320 patients were diagnosed with bronchiolitis between November 2019 and January 2020 (see table 1). The weekly hospital LOS, weekly number of CXRs and blood tests, and weekly representation rates were tracked on run charts.

Initially, there was a decrease in unnecessary investigations in the first week of November 2019: there were no inappropriate X-rays performed, and only $9 \%$ of patients had inappropriate blood tests performed. This was an improvement when compared with the same week in 2018, where there had been $15 \%$ of inappropriate X-rays and $23 \%$ of inappropriate blood tests performed (see figures 1 and 2).

There was a decrease in progress in the number of X-rays in the week of 22 November 2019. The amount of patients having X-rays increased to $24 \%$. After the PDSA cycle and corresponding interventions in the week of 29 November 2019, the number of patients having X-rays were $16 \%$, with $19 \%$ having blood tests. In the week of 6 December 2019, the number of patients undergoing X-rays was $28 \%$ and the number undergoing blood tests 
SPC Chart: Winter $18 / 19$ vs Winter19/20 Weekly Percentage of Bronchiolitis Patients who had Chest X-Ray

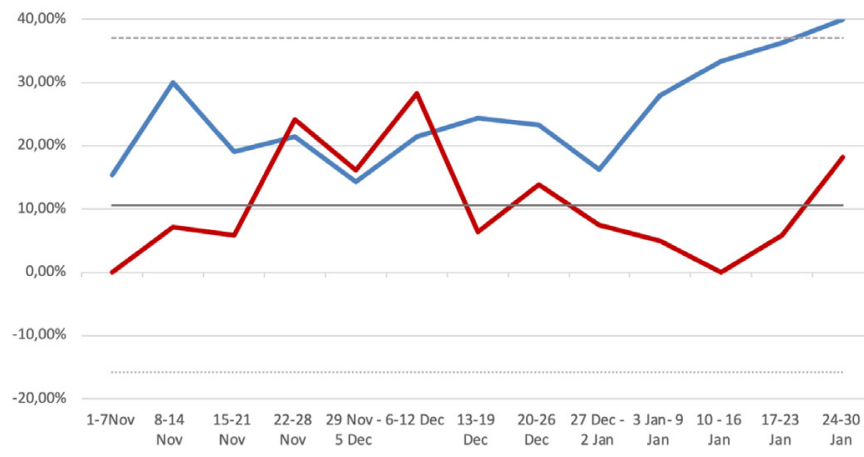

Winter 19/20 Winter 19/20 mean - Winter 19/20 UCL w W... Winter 19/20LCL

Figure 1 SPC chart: winter 2018/2019 vs Winter 2019/2020 weekly percentage of patients with bronchiolitis who had chest X-ray. SPC, statistical process control. UCL, upper control limit. LCL, lower control limit.

was $24 \%$. Finally, by the week of 13 December 2019, numbers had improved, with $6 \%$ of patients having blood tests and X-rays. (See figures 1 and 2)

There was an isolated peak in the number of blood tests performed, in the week starting 3 January 2020, with $25 \%$ of patients having blood tests. Despite this peak, the cumulative average weekly number of blood tests performed was $13 \%$ (see figure 2 ).

Another peak in the number of X-rays performed can be observed in the week starting 24 January 2020, with $18 \%$ of patients undergoing X-rays but with a cumulative average weekly number of X-rays of $11 \%$. (See figure 1 )

Overall, there was a $57 \%$ reduction in the cumulative average weekly CXR rate (from $25 \%$ to $11 \%, \mathrm{p}<0.05$ at 0.009974 ), and there was an improvement of $56 \%$ in the cumulative average weekly laboratory investigation rate (from $29 \%$ to $13 \%, \mathrm{p}<0.05$ at 0.005475 ).

The bronchiolitis presentations to the ED and bronchiolitis admissions to the ward follow a very similar trend in the winter of 2018/2019 and the winter of 2019/2020. It was noted that there was a peak in the week of 6-12

Winter $18 / 19$ vs Winter19/20 Weekly Percentage of Bronchiolitis Patients who had Bloods Taken

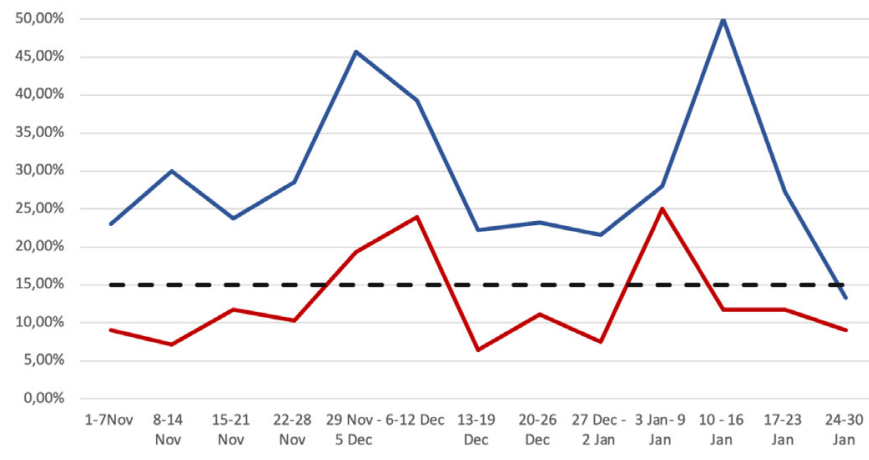

—Winter 18/19 W Winter 19/20 - - target< $15 \%$

Figure 2 Winter 2018/2019 vs Winter 2019/2020 weekly percentage of bronchiolitis.
December 2019 that was not seen in the previous season. The hospital and ED LOS remained similar in both seasons; the average hospital LOS improved postintervention (20.7 hours) compared with preintervention (25.3 hours). There was a non-statistically significant $18 \%$ reduction in the average weekly LOS ( $p$ value 0.270549 ).

The patient representation rate remained similar after the intervention: 15 patients represented to ED after the protocol was initiated, compared with 17 patients before the intervention. The cumulative average weekly representation rate preintervention was $4 \%$ and remained at $4 \%$ postintervention, with a $\mathrm{p}$ value of 0.737 (not statistically significant).

\section{DISCUSSION}

\section{Summary}

The introduction of an evidence-based protocol successfully reduced the number of inappropriate investigations in infants with bronchiolitis, resulting in a decreased hospital LOS and a stable representation rate. The cumulative average weekly CXR and laboratory investigation rate decreased by more than half from the previous season. On review of the postintervention audit of CXRs and laboratory investigations performed, both measures persistently maintained a cumulative average below the target aim of $15 \%$.

Initially, after the introduction of the protocol and an education session among the staff, results were very promising, seen with a decrease in unnecessary investigations in the first week of November 2019.

There was a decrease in progress in the number of X-rays in the week of 22 November 2019. The number of patients having X-rays increased to $24 \%$. Although the cumulative weekly average of X-rays at that time was $9 \%$, well below the $15 \%$ goal, it was still concerning as there was an upward trend in the number of investigations performed. Stakeholder meetings identified that this may be due to new staff on the hospital winter initiative staffing programme who were not accustomed to the protocol.

As a response, education sessions were increased to daily sessions among all hospital staff involved with these patients in an attempt to improve compliance of all staff on all shifts. Aspects of the protocol were elaborated on and also taught in the form of skill sessions, such as the set-up of heated humidified high-flow nasal prong oxygen, insertion of nasogastric tubes and calculation of nasogastric feeds. This improved the team's confidence in the use of the protocol. This took 3 weeks to truly have an impact. After this corresponding intervention, the number of patients undergoing investigations improved so that, by the week of 13 December 2019, only $6 \%$ of patients had blood tests and X-rays.

After the unexplainable peak in inappropriate blood tests from 3 January 2019, where $25 \%$ of patients had blood tests, posters were put up as reminders about the protocol in the workstations. 
Another peak in inappropriate X-rays performed in the week starting 24 January necessitated electronic reminders in the form of emails that encouraged staff to follow the protocol.

These isolated peaks can be attributed to the nature of shift work: if one member of staff does not have confidence in the protocol and that member of staff works in the paediatric ED that week, it affects the week's statistics. As soon as a new shift pattern starts, the numbers drastically improved again in the following week. Despite these peaks in the numbers of inappropriate investigations, the average total weekly number of investigations remained below the goal of $15 \%$ and below the number found in the preintervention audits the previous year.

To our knowledge, this is the largest audit on inappropriate investigations in bronchiolitis infants in Ireland to date.

\section{Interpretation}

This project can be compared with a quality improvement project done by Ralston et al:

'A Multicentre Collaborative to Reduce Unnecessary Care in Inpatient Bronchiolitis'. ${ }^{8}$ They used a 'voluntary collaborative' through webinars that occurred monthly, between 21 participating hospitals in the USA, to reduce inappropriate care in these hospitals for bronchiolitis children under 24 months of age, comparing two seasonsin 2013 and 2014. They were successful in reducing LOS by 5 hours; CXRs decreased by $44 \%$; and readmissions were unchanged. ${ }^{8}$ By using an evidence-based protocol with collaboration sessions through teaching, our rate of CXRs decreased even more by $57 \%$.

This project had a positive impact on the ED. Feedback at stakeholder meetings was that the new protocol flowchart was easy to use, enabled appropriate escalation of ill patients and appropriate discharge of patients with mild disease. Other feedback also stated that doctors were more confident in their decisions about patient severity and treatment, without wasting time and resources with inappropriate investigations.

\section{Limitations}

There was no measure of the patient's perspective of quality of care, in the form of a questionnaire, etc, and this is a valid aspect to consider.

Accuracy of the ED or hospital LOS is dependent on documented time of discharge as written by the treating clinician. The absence of an electronic medical record system may also lead to further measurement error.

Patients who were triaged under a category other than respiratory distress will not have been identified during the data collection.

\section{CONCLUSION}

This was a project that had a profound impact on the quality of care in the paediatric ED, as it addressed a patient population that has a high burden on the ED. During the winter months, there are a large number of infants with bronchiolitis in the ED, and a streamlined, evidence-based protocol has been proven to be effective in facilitating effective management and decision making regarding best care of these patients.

To ensure that the change is not a temporary change, the new protocol has formed a part of the hospital guideline. New doctors who start their rotation through Cork University Hospital undergo an orientation lecture on the topic and the protocol.

Nursing education had enabled there to be a permanent member of staff familiar with the hospital protocol on every shift, to guide any new or temporary members of the team in the right direction towards the aim. The protocol is easy to access on all the hospital computers and in the form of posters in the paediatric ED and the resuscitation unit.

It is important to emphasise that this protocol is only effective with diligent reinforcement and education. In order for the protocol to be reproducible, the team involved needs to be educated and trained in the clinical skills required.

Other interventions that may prove useful to further decrease the number of CXRs and blood tests performed on these patients should be explored; for example, a pop-up reminder on the electronic system when investigations are ordered to remind a physician that a diagnosis of bronchiolitis should not rely on these investigations.

A more accurate way to record time to patient disposition should be sought, to accurately determine whether this protocol facilitates the doctor's decision making.

Future audits should also assess for any inappropriate treatments of children with bronchiolitis, which should not occur when this protocol is adhered to.

The effectiveness of nasogastric as opposed to intravenous rehydration in these patients could potentially inspire other protocols to be created for nasogastric rehydration in infants with, for example, gastroenteritis.

This project had succeeded to place an emphasis on the importance and efficacy of good clinical examination above other investigations, a skill that is almost lost in the busy ED where investigations such as X-rays and blood tests have become a culture in the work-up of an unwell patient. The use of an evidence-based protocol with diligent teaching uplifts this skill and will ensure best quality of patient care.

Acknowledgements We thank the staff of Cork University Hospital for their support in this project and their dedication to quality improvement.

Contributors LJ, G-PM and ROB: conceptualisation, data collection, analysis, writing and editing; $\mathrm{GC}$ and $\mathrm{BL}$ : data collection. $\mathrm{LJ}$ is the guarantor. ROB is the supervising consultant.

Funding The authors have not declared a specific grant for this research from any funding agency in the public, commercial or not-for-profit sectors.

Competing interests None declared.

Patient consent for publication Not required.

Provenance and peer review Not commissioned; externally peer reviewed. 
Data availability statement All data relevant to the study are included in the article or uploaded as supplementary information.

Supplemental material This content has been supplied by the author(s). It has not been vetted by BMJ Publishing Group Limited (BMJ) and may not have been peer-reviewed. Any opinions or recommendations discussed are solely those of the author(s) and are not endorsed by BMJ. BMJ disclaims all liability and responsibility arising from any reliance placed on the content. Where the content includes any translated material, BMJ does not warrant the accuracy and reliability of the translations (including but not limited to local regulations, clinical guidelines, terminology, drug names and drug dosages), and is not responsible for any error and/or omissions arising from translation and adaptation or otherwise.

Open access This is an open access article distributed in accordance with the Creative Commons Attribution Non Commercial (CC BY-NC 4.0) license, which permits others to distribute, remix, adapt, build upon this work non-commercially, and license their derivative works on different terms, provided the original work is properly cited, appropriate credit is given, any changes made indicated, and the use is non-commercial. See: http://creativecommons.org/licenses/by-nc/4.0/.

\section{ORCID iDs}

Lizeri Jansen http://orcid.org/0000-0001-6338-365X

Gideon-Phil Meyer http://orcid.org/0000-0001-7608-7657

Glenn Curtin http://orcid.org/0000-0002-2182-2340

Rory 0'Brien http://orcid.org/0000-0001-9588-7801

\section{REFERENCES}

1 Pelletier AJ, Mansbach JM, Camargo CA. Direct medical costs of bronchiolitis hospitalizations in the United States. Pediatrics 2006;118:2418-23.

2 Florin TA, Byczkowski T, Ruddy RM, et al. Variation in the management of infants hospitalized for bronchiolitis persists after the 2006 American Academy of pediatrics bronchiolitis guidelines. $J$ Pediatr 2014;165:786-92.

3 National Institute for health and Care and Excellence (NICE). Bronchiolitis in children: diagnosis and management [internet]. Available: https://www.nice.org.uk/guidance/ng9

4 Royal Children's Hospital (RCH). RCH clinical practice guidelines bronchiolitis, 2017. Available: https://www.rch.org.au/clinicalguide/ guideline_index/Bronchiolitis/

5 O'Brien S, Borland ML, Cotterell E, et al. Australasian bronchiolitis guideline. J Paediatr Child Health 2019;55:42-53.

6 Children's Hospital of Philadelphia (CHOP). CHOP bronchiolitis clinical pathway, 2019. Available: https://www.chop.edu/clinical-pathway/ bronchiolitis-clinicalpathway- respiratory-assessment

7 Irish Association for Emergency Medicine (IAEM). IAEM paediatric clinical guideline bronchiolitis version 1, 2018. Available: http:// www.iaem.ie/wpcontent/uploads/2019/04/IAEM-CG-Bronchiolitis. pdf

8 Ralston SL, Garber MD, Rice-Conboy E, et al. A multicenter collaborative to reduce unnecessary care in inpatient bronchiolitis. Pediatrics 2016;137:2015-0851. 